\title{
Adaptation to field displacement during head movement unrelated to the constancy of visual direction
}

\author{
HANS WALLACH, KARL JOSEF FREY, AND GEORGE ROMNEY 2 \\ SWAR THMORE COLLEGE
}

\begin{abstract}
Adaptation to vertical field displacements dependent on head turning about a vertical axis was demonstrated under two conditions, rapid training with 100 head movements and 1-h-long training with continuous head turning. The effect of rapid training was measured with the slant estimation method. Adaptation after the longer training was ascertained by comparing the uncertainty ranges for apparent target immobility before and after the adaptation period. Adaptation to field displacements in directions parallel to the plane of the head rotation obtained under corresponding conditions was also measured and found to be somewhat greater than adaptation to vertical field displacements. The result of work by Wallach and Frey that adaptation to field displacement in the direction with the head rotation is greater than to displacement against it was corroborated. While the previous result had.been obtained with rapid adaptation and with the slant estimation method, we confirmed it with 1-h training and by measuring the uncertainty ranges before and after the adaptation period.
\end{abstract}

When the visual field is displaced relative to an S's head causing image displacement across the retina or pursuit movements of the eyes, the field is seen to move. But when the same kind of displacement causing similar stimulus processes is produced by a head movement of $\mathrm{S}$, the field appears stationary. This is the result of a compensating process where the head movement is taken into account in the evaluation of displacements between the visual field and the head. We deal here with a perceptual constancy akin to size constancy, etc. This constancy of visual direction, as Wallach and Kravitz (1965a) have called it, operates with great accuracy; even small objective displacements of the visual field during head movements, which would increase or diminish the normal head-movement-caused displacement of the field relative to the head, will be perceived as field displacements. In other words, field displacements during head turning in the amount of the head rotation but in the direction opposite to it will be perceived as field immobility, while larger or smaller field displacements will be perceived as field motion. Field motion in the direction against the head movement direction will be perceived when the given field displacement is larger than normal and perceived field motion will be in the direction with the head movement when the given field displacement is smaller than normal.

Previous work (Wallach \& Kravitz, 1968) has shown that this compensating function can be easily modified. Partial adaptation to consistent abnormal field displacements can be readily produced. When $S$ is exposed for some time to conditions that cause field displacements that are, say, smaller than normal in a certain amount, below-normal field displacements of some value can be found that lead to perceived field immobility, while normal field displacement will be perceived as field displacement in the direction against the head turning. The latter fact can be used as a qualitative test of adaptation, while finding the particular $a b$ normal field displacement that is perceived as field rest measures the adaptation effect.

Stated in practical terms, normal field displacement during head movements is, of course, produced by objective field immobility, the normal condition of our lives. Below-normal and above-normal field displacements are produced by causing the visual field to be displaced during head movements and dependent on them. This can be done optically by equipping Ss with minifying or magnifying spectacles or by mechanical means (Wallach \& Kravitz, 1968). In the latter case the field is represented by a dimly luminous target spot in an otherwise dark environment. This target is projected via a reflecting mirror and will become displaced when the mirror is turned. Head turning is transferred to the mirror by means of a variable transmission, enabling $\mathrm{E}$ to present objective target motion in the direction with or against the head movement in any desirable ratio to the head turning. In order to represent below-normal or above-normal field displacement, such objective target displacement must be parallel to the plane of the rotation in which the head movement consists, and this is true of the mechanical device used so far in such experiments. It is true also of optical field displacements produced by power lenses.

The present report is concerned with adaptation to objective target displacements perpendicular to the plane of the head rotation rather than parallel to it. Inasmuch as in ordinary life such displacements between field and head never occur, adaptation to vertical target displacement would be unrelated to the constancy of visual direction. We demonstrated such adaptation in two ways: by rapid adaptation measured with the slant estimation technique developed by Wallach and Frey (1969) and by 1-h-long adaptation measured by Wallach and Kravitz' method (1968) of determining no-motion ranges.

\section{RAPID ADAPTATION TO UNRELATED} \section{TARGET DISPLACEMENT DURING HEAD MOVEMENTS ${ }^{3}$}

Adaptation was produced here by having $S$ in a dark room observe a luminous target spot that moved up and down when $S$ turned his head to the right and the left. The vertical displacement angle of the target was $40 \%$ of the angle of the head rotation. The measuring device used by Wallach and Kravitz (1968) and equipped with two mirrors by Wallach and Frey (1969) was employed. To create the adaptation conditions, the vertically displacing mirror was connected to the input shaft while the mirror that caused the horizontal target displacement was arrested.

Preliminary experiments had shown that, after an $S$ had observed for some time a target being displaced upward when his head turned right and downward when his head turned left, a stationary target would appear to move down when the head turned right and up when it turned left. We made this adaptation-caused apparent vertical target motion measurable in a manner corresponding to that used by Wallach and Frey, namely, by transforming the extent of the apparent vertical target motion into the slant of an apparent oblique motion path. This was done by giving the stationary target spot an objective horizontal displacement of $40 \%$ of the head rotation. The adaptation-caused apparent vertical displacement added itself vectorially to the objectively given horizontal target displacement, resulting in an oblique apparent motion path, whose slant could be reproduced by $S$. This was done with the help of a metal rod attached at its midpoint to a horizontal 
shaft that turned in a bearing inserted in a large white board. The board was placed near $\mathrm{S}$, somewhat to the side so that it did not obscure the target's horizontal motion path. The board and the plane in which the rod turned were in frontal-parallel position after $S$ had turned his head toward it, and the shaft was at S's eye level. In reproducing the slant of the apparent target motion $S$ turned the rod with his hand.

\section{Procedure}

There were $16 \mathrm{Ss}$ and each participated in two experimental sessions at least 2 days apart. Each session began with a test in which $S$, sitting under the device previously used by Wallach and Frey, his head attached to the input shaft, had to turn his head toward the rod and set it to the horizontal. Then, with the vertically displacing mirror disconnected and the transmission set for the other mirror to cause a horizontal target displacement of $40 \%$ of the head rotation, S made three head movements and reproduced the direction of the target's perceived motion path by a rod setting. $S$ made two such settings, one with the horizontal target in the direction with the head movement and the other against it. This was done because after adaptation also two tests were made that differed in the direction of the horizontal displacement.

Adaptation training consisted in 100 complete head movements during which the target moved vertically always upward when the head turned to the right and downward with left turning. The ratio of target displacement to the head rotation angle was $40 \%$. When the 100 head movements were completed, which took about $3 \mathrm{~min}$, the vertically displacing mirror was again disconnected and the other mirror set to produce an objective horizontal target displacement with the head movement. $\mathrm{S}$ was allowed three head movements during which he observed the slant of the apparent motion path and then reproduced that slant with a rod setting. Immediately thereafter the direction of the objective horizontal target displacement was reversed and, after another three head movements, $\mathrm{S}$ made another rod setting, now for horizontal displacement against the head movement. The difference in slant angle between corresponding pre- and postadaptation settings represents the adaptation effect. The second experimental session differed from the one just described only in that the horizontal target displacement in the first test was against the head movement and in the second test in the with direction.

\section{Results}

The mean adaptation effects are listed in Table 1 under the heading "first adaptation test." Amounting to $6 \mathrm{deg}$, they approach the effects that Wallach and Frey (1969) obtained under very similar conditions for adaptation to target displacement parallel to the plane of the head rotation. The effects were highly significant as shown by the confidence limits also given in Table 1 . The effect disappears rapidly as demonstrated by the immediately following second test, which produced settings of significantly smaller slants. Since in a test the target spot does not undergo vertical displacement-the horizontal displacement of the target does not alter the matter in principle-the test represents normal viewing conditions and is therefore an occasion that can cause undoing of the adaptation to the artificial displacement conditions of the experiment. A corresponding effect was obtained by Wallach and Frey in connection with adaptation to displacements parallel to the plane of the head rotation.

\section{PROLONGED ADAPTATION TO FIELD DISPLACEMENTS PERPENDICULAR TO THE PLANE IN WHICH THE HEAD TURNS}

Adaptation to target displacement during head movements that is unrelated to the constancy of visual direction is so important a fact that we also demonstrated it with an hour-long adaptation period of continuous head movements. This method yields a stabler effect and therefore allows measurement of an uncertainty range. This is the range of ratios of target displacement to head rotation angle over which the target may appear stationary. Its shift after the adaptation period measures the adaptation effect, but by its width it also tells us something about the nature of the effect. Since power lenses previously used for prolonged adaptation periods cause only field displacement parallel to the plane of head rotation, vertical field displacement dependent on head turning was produced by a moving-mirror device. It could be changed to yield horizontal field displacement also, and we were thus able to produce adaptation to constancy-related field displacements, in the directions with and against the head movement as well as vertical field displacements under otherwise identical conditions. In the case of all three adaptation experiments, the displacement rate amounted to $50 \%$ of the angular displacement of the head.

\section{Apparatus}

In the device used for adaptation training, $S$ sat under a horizontal plate in which a vertical shaft was mounted. A thin vertical plate fixed in median position to the top of S's headgear could be inserted into the slotted end of this shaft. S's head could be adjusted to meet the shaft by raising the chair on which he sat. Three sets of angle gears and two additional shafts transmitted the rotation of the vertical shaft caused by S's head turning to a mirror mounted on a horizontal shaft. The mirror was close to S's face at the level of his eyes. It was slanted to form a 45-deg angle with the vertical when S's head pointed straight forward, and it was under mild spring tension to take up the play in the various gears. A second larger, stationary mirror, also slanted approximately $45 \mathrm{deg}$ and its reflecting surface facing the movable mirror, permitted $S$ to see a television set located straight ahead of him at a distance of $3.5 \mathrm{~m}$. The stationary mirror was so adjusted that $S$ saw the set straight ahead at eye level when his head pointed forward. This arrangement caused the set to be shifted optically up and down when $S$ turned his head back and forth. Two gear arrangements were provided, one which caused the set to shift optically up when $S$ turned his head to the right and the other for the set to appear to

Table 1

Mean Adaptation to Vertical Target Displacement in Degrees of Slant after 100 Head Movements

\begin{tabular}{|c|c|c|c|c|c|}
\hline \multicolumn{2}{|c|}{ First adaptation test } & \multicolumn{2}{|c|}{ Second adaptation test } & \multirow{2}{*}{$\begin{array}{l}\text { Difference } \\
\text { between first } \\
\text { and second test }\end{array}$} & \multirow[b]{2}{*}{$\mathrm{p}$} \\
\hline $\begin{array}{l}\text { Direction of } \\
\text { horizontal } \\
\text { displacement }\end{array}$ & $\begin{array}{l}\text { Adaptation } \\
\text { effect; confi- } \\
\text { dence limits } \\
\text { at } .05 \text { level }\end{array}$ & $\begin{array}{l}\text { Direction of } \\
\text { horizontal } \\
\text { displacement }\end{array}$ & $\begin{array}{l}\text { Adaptation } \\
\text { effect }\end{array}$ & & \\
\hline With & $6.06 \pm 1.76$ & Against & 4.53 & 1.53 & $<.02$ \\
\hline Against & $6.03 \pm 1.73$ & With & 4.87 & 1.16 & $<.02$ \\
\hline
\end{tabular}


move down for the same turn direction. Always, the mirror turned at a rate of $25 \%$ of that of the head rotation, causing the set to become optically displaced at a rate of $50 \%$ of the head rotation angle.

To provide horizontal field displacement, the device was changed so that the movable mirror turned on a vertical shaft, making $S$ see the TV set through a stationary mirror located at his side. The turning rate of the mirror remained the same and the ratio of the now lateral optical displacement of the set was again $50 \%$. Apart from the mirrors, all surfaces of the device visible to $\mathrm{S}$ were masked with black tape. Except for a flashlight sparingly used by $E$, the room was kept dark throughout the experiment. The TV picture was dimmed to the point where its light was restricted to its immediate environment that was visible only in the mirror. Therefore, only those objects were seen during the adaptation period that underwent the appropriate displacements when $\mathrm{S}$ moved his head.

To count head movements, a rod was fixed at right angles to the vertical shaft to which S's headgear was attached. The end of this rod, which thus moved through an arc when $S$ turned his head, operated two switches, one at each end of its motion path. An arrangement of relays tripped an electric counter each time both switches had been closed successively in one order and thus caused only completed movement cycles to be counted. The switches were so spaced that $S$ had to turn his head through $30 \mathrm{deg}$ in order to activate both of them, and they provided stops that limited the head movements to 36 deg.

The measuring device used to determine the uncertainty ranges before and after the adaptation period was described in detail by Wallach and Kravitz (1968). It caused a circular target spot to become displaced horizontally when $\mathrm{S}$ turned his head. The displacement ratio, that is, the rate of target displacement relative to the head rotation, was variable and could be set by $E$, and the target spot could be made to shift in the direction with the head rotation or against it. In the work here reported we used this device to measure adaptation to horizontal field displacement.

To measure adaptation to vertical field displacement, the device was altered: The mirror that reflected the target spot was replaced by one that rotated about a horizontal shaft when $S$ turned his head, causing vertical displacement of the target spot. Again, its displacement ratio was variable and the direction of the displacement of the target spot was also alterable; it could be upward for a turning of the head to the right, or downward.

\section{Procedure}

Adaptation was measured by obtaining the uncertainty range of no-motion judgments before and after the adaptation period and by computing the difference of their midpoints. The manner of measuring the uncertainty range was similar to that previously employed and explained in the article by Wallach and Kravitz (1968). Again the rate of target displacement relative to the head rotation, the displacement ratio (DR) was varied. Where adaptation was to constancy related field displacement, that is, to displacement in the direction with or against the head rotation, one series of displacement ratios was presented that led, in that order, to perceived target displacement in the direction with the head movement, to apparent target rest, and to perceived target motion against the head rotation. The other series of trials covered the relevant part of the scale of displacement ratios in the opposite order. In the preadaptation measurements, the part of the DR scale presented comprised objective target immobility as well as displacement with and against the head movement. After adaptation had developed, causing target displacement of a certain DR range to be perceived as stationary, the trials to be presented were, of course, centered about this range.

Trials were spaced in steps of $.8 \% \mathrm{DR}$, and the sequence of trials was presented twice, once in each direction, yielding altogether two limits at each end of the no-motionrange. As in the previous work the uncertainty range was computed by plotting from the two sequences those trials that yielded no-motion reports or inconsistent ones; one-half step was added at each end of the range. When adaptation was to field displacements against the head movement-causing a range of displacement ratios against the head rotation to lead to perceived target rest-the first postadaptation sequence started with target displacement against the head movement of high DR leading to perceived target motion in that direction. This was done because the presentation of displacements leading to perceived target motion with the head rotation tends to diminish the established adaptation. By probing for the relevant DR range with trials yielding target motion against the head, the presentation of trials causing perceived target motion with the head was minimized. In the case of adaptation to displacement with the head rotation, the procedure was, of course, reversed. To give $S$ practice with these measurements, the preadaptation test was preceded by two trial sequences each of which determined only one of the two limits of the no-motion range, i.e., did not continue across the no-motion range to find the second limit, as happened in the subsequent actual test.

Adaptation to vertical field displacement was tested in a corresponding manner. Thus, there were two directions of vertical target displacement relative to the head rotation, one upwards when S's head turned right and downward when it turned left, and the other with reversed target displacements.

Subjects spent $1 \mathrm{~h}$ in the training device and made from 992 to 3046 complete head movements during that period. The same $12 \mathrm{Ss}$ were at different times employed in all three conditions of the experiment. The order of adaptation to field displacements with or against the head rotation was randomized. In the case of vertical field displacement, six Ss adapted to upward displacement when the head turned right and the other six to relative displacement in the opposite direction.

\section{Results}

As in our first experiment, adaptation to vertical field displacement yielded a sizable effect. The mean difference between the midpoints of the uncertainty ranges measured before and after 1-h adaptation amounted to $8.73 \% \mathrm{DR}$, and this effect was highly significant. There was no overlap of the uncertainty ranges taken before and after adaptation for any one of our Ss. As was to be expected, this effect is greater than the one obtained from a brief training period in our first experiment. The mean change in slant estimate in the amount of $6 \mathrm{deg}$ obtained there was equivalent to $4.2 \%$ DR. The difference between the two adaptation effects appears small when one considers that the large one results from 10 times more training than the smaller one. The comparatively small effect obtained after the long training of our second experiment cannot be attributed to a loss in adaptation gradually taking place during the much longer measuring procedure here employed. Such a progressive loss would have manifested itself in an enlarged uncertainty range measured after adaptation. The mean uncertainty ranges measured after adaptation show that no important enlargement occurred (Table 2).

The second experiment permits comparing adaptation to vertical field displacement with adaptation to constancyrelated field displacement produced under closely similar conditions. For both conditions of horizontal field displacement mean adaptation was greater than adaptation to vertical 
Table 2

Adaptation to Field Displacement in Three Directions Compared All Means in \% DR

\begin{tabular}{|c|c|c|c|c|c|}
\hline \multirow{2}{*}{$\begin{array}{l}\text { Relative field } \\
\text { displacement }\end{array}$} & \multicolumn{2}{|c|}{ Uncertainty Range } & \multicolumn{2}{|c|}{ Mean Adaptation } & \multirow[b]{2}{*}{$p$} \\
\hline & Before & After & Effect & Difference & \\
\hline Vertical & 2.39 & 2.65 & 8.73 & & \\
\hline $\begin{array}{l}\text { With head } \\
\text { rotation }\end{array}$ & 4.17 & 4.04 & 14.69 & 5.96 & $<.01$ \\
\hline $\begin{array}{l}\text { Against head } \\
\text { rotation }\end{array}$ & 3.31 & 4.51 & 10.14 & 4.55 & $<.03$ \\
\hline
\end{tabular}

displacement, but a significant difference was obtained only in the case of adaptation to displacement in the direction with the head rotation $(p<.01)$. The mean uncertainty ranges for horizontal target displacement were larger than for vertical target displacement, both before and after adaptation. With the data for the two horizontal conditions combined, each difference was significant at the .05 level on two-tailed tests.

When the two constancy-related adaptation effects are compared with each other one finds that they confirm the result of Wallach and Frey (1969) who found that adaptation to field displacement with the head rotation was consistently greater than that to displacement in the opposite direction. We obtained a significant difference in the same direction $(4.55 \%$ DR, $\mathrm{p}<.03)$. $^{4}$

\section{DISCUSSION}

The result of our work that partial adaptation to field displacements in an unrelated direction can be obtained as easily as adaptation to constancy-related displacements is confirmed by an experiment of John Hay (1968), conceived independently from ours. Employing an electronic technique he had Ss adapt to horizontal displacements of a target point that were dependent on a nodding head movement, that is, on head rotation about a horizontal axis. Translated into the terminology used by us Hay's one quantitative experiment had the following result: Ten minutes of adaptation to horizontal target displacements of $70 \%$ DR when head movements were vertical yielded a mean adaptation of $8.4 \% \mathrm{DR}$; eight Ss participated each of whom made a single no-motion setting, that is, one setting that fell somewhere within the uncertainty range. 5

There are two ways in which to account for the existence of a learned constancy such as the constancy of visual direction or, more generally speaking, of instances of perceptual adaptation. They may be due to specific learning capacities, each existing to meet its particular purpose. The assumption that they are based on specific learning processes would particularly fit such adaptive capacities that are necessary to compensate for normal changes of the body as growth of the skull (causing increase in interocular distance and hence in retinal disparity ${ }^{6}$ ) or changes in ocular optics that produce shape distortions in the visual field. It could, however, also apply to such seemingly vital functions as the constancy of visual direction. Alternatively, some adaptation processes may be manifestations of general principles that can underlie a variety of cases of perceptual learning. ${ }^{7}$ The finding that adaptation to field displacements unrelated to the constancy of visual direction can be obtained at a magnitude comparable to constancy-related adaptation favors the latter view.

In constancy-related adaptation, an adaptation to the normal field displacements relative to the turning head that $S$ brings to the experiment, is modified; adaptation is to a condition where the normal displacements of an objectively stationary field are either augmented or diminished. But when the objective field displacement is vertical, the normal horizontal field displacement is thereby made oblique, a direction that is the result of the two displacement components. (In our second experiment, for instance, where the objectively vertical field displacement amounted to $50 \%$ of the rate of head rotation this oblique displacement formed an angle of $26.6 \mathrm{deg}$ with the horizontal.) The adaptation effect that results, however, does not consist in a modification of normal adaptation. Rather, $S$ retains the adaptation to the normal, head-rotation-caused field displacement and develops an apparently independent adaptation to vertical field displacement. This is indicated by two facts: After the adaptation period, an objectively stationary target spot appears to move vertically, and the uncertainty range for vertical target displacement remains small after adaptation has taken place, significantly smaller than that measured for horizontal target displacement. This independent adaptation to vertical field displacement is unique in kind in S's life; never before was he exposed to the combination of vertical field displacement occurring with head rotation in the horizontal plane. Yet he easily and rapidly adapts to these conditions and this argues that some general principle is in operation here rather than a specific learning capacity related to the field displacements normally caused by head movements and responsible for the constancy of visual direction.

\section{REFERENCES}

HAY, J. Visual adaptation to an altered correlation between eye movement and head movement. Science, 1968, 160, 429-430.

WALLACH, H., \& FREY, K. J. Adaptation in the constancy of visual direction measured by a one-trial method. Perception \& Psychophysics. 1969, 5, 245-252.

WALLACH, H., \& KARSH, E. The modification of stereoscopic depth-perception and the kinetic deptheffect. American Journal of Psychology, 1963, 76, 429-435.

WALLACH, H., \& KRAVITZ, J. The measurement of the constancy of visual direction and of its adaptation. Psychonomic Science, 1965a, 2, $217-218$

WALLACH, H., \& KRAVITZ, J. Adaptation in the constancy of visual direction tested by measuring the constancy of auditory direction. Perception \& Psychophysics, 1968, 4, 299-303.

WALLACH, H., MOORE, M., \& DAVIDSON, L. Modification of stereoscopic depth-perception. American Journal of Psychology, 1963, 76, 191-204.

\section{NoTES}

1. The work was supported by Grant 11089 by the National Institutes of Health to Swarthmore College, Hans Wallach principal investigator.

2. Address: Department of Psychology, Swarthmore College, Swarthmore, Pennsylvania 19081.

3. This experiment was first reported at a colloquium of the Department of Psychology of Harvard University in April, 1967.

4. An interpretation of this finding is presented in Wallach and Frey (1969).

5. Hay attributes adaptation to head movement-dependent object displacement to the occurrence of eye movements that follow the displacing object during the adaptation process. He supports this view by an experiment in which a stationary as well as a displacing object are visible during the adaptation exposure. He found an adaptation effect such that a stationary object appeared to move when $\mathrm{S}$ nodded his head when during the adaptation exposure $S$ looked at the displacing object, but no such aftereffect occurred when $S$ fixated the stationary object. This outcome can be interpreted in a different way: the two objects provide conflicting information and selective attention causes the different outcomes of the adaptation exposure. The absence of measurements that show that adaptation is as great in the presence of the stationary object as without it makes this demonstration inconclusive.

6. Rapid partial adaptation of stereoscopic depth perception to artificially altered binocular parallax, which corresponds to change in interocular distance, was demonstrated by Wallach, Moore and Davidson (1963).

7. An example is the hypothesis of process assimilation which would account for adaptation that takes place when one of a pair of cues giving tise to the same perceptual property is artificially altered. This hypothesis was first proposed by Wallach and Karsh (1963) in connection with adaptation of stereoscopic depth perception. A more detailed discussion may be found in Chapter 13 of The neuropsychology of spatially oriented behavior, 1968, Sanford J. Freedman, editor.

(Accepted for publication December 24, 1968.) 